PROCEEDINGS OF THE

AMERICAN MATHEMATICAL SOCIETY

Volume 133, Number 12, Pages 3597-3607

S 0002-9939(05)08163-3

Article electronically published on June 8, 2005

\title{
SAMPLING EXPANSIONS FOR FUNCTIONS HAVING VALUES IN A BANACH SPACE
}

\author{
DEGUANG HAN AND AHMED I. ZAYED
}

(Communicated by David R. Larson)

\begin{abstract}
A sampling expansion for vector-valued functions having values in a Banach space, together with an inversion formula, is derived. The proof uses the concept of framing models of Banach spaces that generalizes the notion of frames in Hilbert spaces. Two examples illustrating the results are given, one involving functions having values in $L^{p}[-\pi, \pi], 1<p \leq 2$, and the second involving functions having values in $L^{p}(\mathbb{R})$ for $1<p<\infty$.
\end{abstract}

\section{INTRODUCTION}

The Whittaker-Shannon-Kotel'nikov (WSK) sampling theorem states that if a function $f$ is band-limited to $[-\sigma, \sigma]$, i.e., it is representable as

$$
f(t)=\int_{-\sigma}^{\sigma} e^{-i x t} g(x) d x \quad(t \in \mathbb{R})
$$

for some function $g \in L^{2}(-\sigma, \sigma)$, then $f$ can be reconstructed from its samples, $f(k \pi / \sigma)$, that are taken at the equally spaced nodes $k \pi / \sigma$ on the time axis $\mathbb{R}$. The construction formula is

$$
f(t)=\sum_{k=-\infty}^{\infty} f\left(\frac{k \pi}{\sigma}\right) \frac{\sin (\sigma t-k \pi)}{(\sigma t-k \pi)} \quad(t \in \mathbb{R})
$$

the series being absolutely and uniformly convergent on $\mathbb{R}$. See, e.g., [17, p. 16].

Because the series in Equation (1.2) can be put in the form

$$
f(t)=\sum_{k=-\infty}^{\infty} f\left(t_{k}\right) \frac{G(t)}{\left(t-t_{k}\right) G^{\prime}\left(t_{k}\right)}
$$

where $t_{k}=k \pi / \sigma$ and

$$
G(t)=\sin \sigma t=\sigma t \prod_{k=1}^{\infty}\left(1-\frac{t^{2}}{t_{k}^{2}}\right)
$$

we shall say that the sampling series is a Lagrange-type interpolation series.

One of the generalizations of the WSK theorem, due to Kramer [11, states that if there exist a function $K(x, t)$ continuous in $t$ such that $K(x, t) \in L^{2}(I)$ as a

Received by the editors November 21, 2003 and, in revised form, July 23, 2004.

2000 Mathematics Subject Classification. Primary 46B15, 46B45; Secondary 94A20, 42 C40.

Key words and phrases. Framing models, Banach spaces, atomic decomposition, interpolation, the Whittaker-Shannon-Kotel'nikov sampling theorem, wavelet basis. 
function in $x$ for every real number $t$ and a sequence of real numbers $\left\{t_{n}\right\}_{n \in \mathbb{Z}}$ such that $\left\{K\left(x, t_{n}\right)\right\}_{n \in \mathbb{Z}}$ is a complete orthogonal family in $L^{2}(I)$ for some finite interval $I=[a, b]$, then for any function of the form

$$
f(t)=\int_{a}^{b} F(x) \bar{K}(x, t) d x=\langle F, K\rangle,
$$

with $F \in L^{2}(I)$, we have $f(t)=\sum_{n=-\infty}^{\infty} f\left(t_{n}\right) S_{n}^{*}(t)$, where

$$
S_{n}^{*}(t)=\frac{\int_{a}^{b} \bar{K}(x, t) K\left(x, t_{n}\right) d x}{\int_{a}^{b}\left|K\left(x, t_{n}\right)\right|^{2} d x} .
$$

It is easy to see that the WSK Theorem is a special case of the Kramer Theorem because if we take $I=[-\sigma, \sigma], K(x, t)=e^{i x t}$, and $t_{n}=\frac{n \pi}{\sigma}$, then $\left\{e^{i t_{n} x}\right\}_{n=-\infty}^{\infty}$ is a complete orthogonal set in $L^{2}(I)$, and in addition

$$
S_{n}^{*}(t)=\frac{\sin \sigma\left(t-t_{n}\right)}{\sigma\left(t-t_{n}\right)}=\operatorname{sinc}\left(\sigma\left(t-t_{n}\right) / \pi\right) .
$$

Kramer [1] noted that the kernel function $K(x, t)$ and the sampling points $\left\{t_{n}\right\}_{n \in \mathbb{Z}}$ may be found from certain boundary-value problems. More precisely, let $L$ be the differential operator defined by

$$
L=p_{0}(x) \frac{d^{n}}{d x^{n}}+\cdots+p_{n-1}(x) \frac{d}{d x}+p_{n}(x), \quad x \in I,
$$

where $p_{k}(x)$ is a complex-valued function with $n-k$ continuous derivatives, $k=$ $0,1, \cdots, n$, on $I=(a, b)$, and $p_{0}(x) \neq 0$ for any $x \in(a, b)$, with $-\infty \leq a<b \leq \infty$. The adjoint operator $L^{*}$ is defined as

$$
L^{*} g=(-1)^{n} \frac{d^{n}}{d x^{n}}\left(\bar{p}_{0} g\right)+(-1)^{n-1} \frac{d^{n-1}}{d x^{n-1}}\left(\bar{p}_{1} g\right)+\cdots+\bar{p}_{n} g .
$$

Let $U_{j}(y)=0, j=1, \cdots, n$, be boundary conditions of the form

$$
U_{j}(y)=\sum_{k=1}^{n}\left(\alpha_{j, k} y^{(k-1)}(a)+\beta_{j, k} y^{(k-1)}(b)\right), \quad j=1,2, \cdots, n,
$$

and consider the boundary-value problem [13, p. 82]

$$
\begin{aligned}
& L y=-t y, \quad x \in I, \\
& U_{j}(y)=0, \quad j=1, \cdots, n .
\end{aligned}
$$

Kramer observed that if the boundary-value problem (1.7) and (1.8) is regular, self-adjoint, and possesses a function $\phi(x, t)$ that generates the eigenfunctions of the problem $\left\{\phi_{n}(x)\right\}$, when the eigenvalue parameter $t$ is replaced by the eigenvalues $\left\{t_{n}\right\}$, i.e., $\phi\left(x, t_{n}\right)=\phi_{n}(x)$, then one can take the sampling points to be $\left\{t_{n}\right\}$ and the kernel function $K(x, t)$ to be $\phi(x, t)$. Any boundary-value problem having that property is said to have the Kramer property. Examples of boundary-value problems having the Kramer property can be found in [17.

It should be emphasized that not every regular, self-adjoint boundary-value problem has the Kramer property. It can be shown that the boundary-value problem: $y^{\prime \prime}=-\lambda y, 0<x<\pi$, with periodic boundary conditions $y(0)=y(\pi), y^{\prime}(0)=y^{\prime}(\pi)$ does not have the Kramer property. 
On the other hand, Kramer's sampling expansion is known to exist for some non-self-adjoint boundary-value problems and even for some self-adjoint problems that do not have the Kramer property. One technique to use for those problems is the Green's function method introduced by A. Zayed in [16]. Under the assumption that the Green's function of the problem, $G(x, \xi, \lambda)$, has only simple poles at the eigenvalues and the boundary conditions are strongly regular (see [1]), we have

$$
G(x, \xi, \lambda)=\sum_{j=1}^{\infty} \frac{\varphi_{j}(x) \bar{\psi}_{j}(\xi)}{\lambda_{j}-\lambda},
$$

uniformly for $x, \xi \in I$, where $\lambda_{n}$ and $\phi_{n}$ are the eigenvalues and eigenfunctions of the problem, and $\psi_{n}$ are the eigenfunctions of the adjoint problem. It is known that $\left\{\phi_{n}\right\}$ and $\left\{\psi_{n}\right\}$ are biorthogonal bases for $L^{2}(I)$.

Now fix $\xi$ and let

$$
\Phi_{\xi_{0}}(x, \lambda):=P(\lambda) G\left(x, \xi_{0}, \lambda\right), \quad \lambda \in \mathbb{C},
$$

where $P$ is an entire function whose zeros are all simple and located exactly at the eigenvalues of the problem. The function $\Phi_{\xi_{0}}(x, \lambda)$ is an entire function in $\lambda$ since the simple poles of $G\left(x, \xi_{0}, \lambda\right)$, i.e., the eigenvalues of (1.7) and (1.8), are cancelled by the simple zeros of $P(\lambda)$. The following theorem was proved in [1] see also [16]. Let $f \in L^{2}(I)$ and $F(\lambda)$ be defined by

$$
F(\lambda)=\int_{a}^{b} \bar{f}(x) \Phi_{\xi_{0}}(x, \lambda) d x .
$$

Then $F(\lambda)$ is an entire function of order $1 / n$ (where $n$ is the order of the differential operator) and type not exceeding $b-a$ that admits the sampling representation

$$
F(\lambda)=\sum_{k=1}^{\infty} F\left(\lambda_{k}\right) \frac{P(\lambda)}{\left(\lambda-\lambda_{k}\right) P^{\prime}\left(\lambda_{k}\right)}, \quad \lambda \in \mathbb{C} .
$$

The sampling series (1.10), which is a Lagrange-type interpolation series, converges uniformly on compact sets of the complex plane and absolutely on $\mathbb{C}$. One can then utilize (1.10) to derive an inversion formula for (1.9) in the form

$$
f(x)=\int_{-\infty}^{\infty} F(\lambda) k(x, \lambda) d \lambda
$$

for some function $k(x, \lambda)$. An extension of these results to Hilbert spaces using frames has been obtained in 15 .

The aim of this article is two-fold: 1) to extend (1.9) to Banach spaces, 2) to derive an analogue of the sampling series (1.10) and the inversion formula (1.11) in a Banach space setting. In order to do that, a notion of frames in Banach spaces is needed.

There are a number of generalizations of frames to Banach spaces. Chief among them are atomic decomposition, Banach frames with respect to sequence spaces, and framing models. Which one to use to derive an analogue of (1.10) and (1.11) was not obvious from the outset. It turns out that framing models are the most suitable for our work. The derivation of the inversion formula uses the sampling theorem, Bochner integrals, and some related inequalities, which fortunately all seem to work nicely with framing models. 
In Section 2 we introduce the notation and preliminary material needed in the sequel. The sampling theorem is given in Section 3 , followed by two examples. The first example deals with functions having values in $L^{p}[-\pi, \pi], 1<p \leq 2$, and the second deals with functions having values in $L^{p}(\mathbb{R})$ for $1<p<\infty$. To the best of our knowledge, these examples are new and do not follow easily from the case $p=2$. The inversion formula is derived in Section 4 .

\section{Preliminaries}

Let $\left\{\lambda_{n}\right\}_{n=1}^{\infty}$ be a sequence of complex numbers, none of which is zero, with the point at infinity as its only limit point. Let us assume that $\lambda_{m} \neq \lambda_{n}$ if $m \neq n$, and let us order them so that $\left|\lambda_{1}\right| \leq\left|\lambda_{2}\right| \leq \cdots$. Assume that there exists an integer $p$ such that $\sum_{n=1}^{\infty} 1 /\left|\lambda_{n}\right|^{p+1}$ converges. Set

$$
G(u ; p)=(1-u) \exp \left(u+\frac{u^{2}}{2}+\cdots+\frac{u^{p}}{p}\right), \quad p \geq 1, \text { and } G(u ; 0)=(1-u)
$$

Then

$$
P(\lambda)=\prod_{n=1}^{\infty} G\left(\frac{\lambda}{\lambda_{n}} ; p\right)
$$

is an entire function in $\lambda$ of order $\tau$, with $p \leq \tau<p+1$, that vanishes exactly at the points $\lambda=\lambda_{n}$ and at no other point. We may include zero as one of the terms of the sequence and replace $P(\lambda)$ by $P_{0}(\lambda)=\lambda P(\lambda)$.

Now we introduce the concept of frames in Banach spaces. Generalizing frames in Hilbert spaces to Banach spaces, C. Gröchenig gave the following definition of atomic decompositions:

Definition 1. Let $X$ be a Banach space and let $X_{d}$ be an associated Banach space of scalar-valued sequences indexed by $\mathbf{N}$. Let $\left(y_{i}\right)$ be a sequence of elements from $X^{*}$ and $\left(x_{i}\right)$ a sequence of elements of $X$. If $(\mathrm{a})\left(\left\langle x, y_{i}\right\rangle\right) \in X_{d}$, for each $x \in X$, (b) the norms $\|x\|_{X}$ and $\left\|\left(\left\langle x, y_{i}\right\rangle\right)\right\|_{X_{d}}$ are equivalent, and (c) $x=\sum_{i=1}^{\infty}\left\langle x, y_{i}\right\rangle x_{i}$, for each $x \in X$, then $\left(\left(y_{i}\right),\left(x_{i}\right)\right)$ is an atomic decomposition of $X$ with respect to $X_{d}$. If the norm equivalence is given by $A\|x\|_{X} \leq\left\|\left(\left\langle x, y_{i}\right\rangle\right)\right\|_{X_{d}} \leq B\left\|_{x}\right\|_{X}$, then $A, B$ are a choice of atomic bounds for $\left(\left(x_{i}\right),\left(y_{i}\right)\right)$.

The idea of general frames actually appeared in 1985 in the work of Frazier and Jawerth [10, where wavelet atomic decompositions were constructed for Besov spaces and were called $\phi$-transforms. After Gröchenig formalized atomic decompositions, there followed a flurry of activity in this area. Feichtinger [6] and Feichtinger and Gröchenig [7, 8, developed a general theory for a large class of function spaces; see also [4, 5]. For a more general setting, Gröchenig 9] gave the following definition.

Definition 2. Let $X$ be a Banach space and let $X_{d}$ be an associated Banach space of scalar-valued sequences indexed by $\mathbf{N}$. Let $\left(y_{i}\right)$ be a sequence of elements from $X^{*}$ and $S: X_{d} \rightarrow X$ be given. If:

(a) $\left(\left\langle x, y_{i}\right\rangle\right) \in X_{d}$, for each $x \in X$,

(b) the norms $\|x\|_{X}$ and $\left\|\left(\left\langle x, y_{i}\right\rangle\right)\right\|_{X_{d}}$ are equivalent,

(c) $S$ is bounded and linear, and $S\left(\left\langle x, y_{i}\right\rangle\right)=x$, for each $x \in X$, 
then $\left(\left(y_{i}\right), S\right)$ is a Banach frame for $\mathbf{X}$ with respect to $X_{d}$. The mapping $S$ is the reconstruction operator. If the norm equivalence is given by

$$
A\|x\|_{X} \leq\left\|\left(\left\langle x, y_{i}\right\rangle\right)\right\|_{X_{d}} \leq B\|x\|_{X}
$$

then $A, B$ are a choice of frame bounds for $\left(\left(y_{i}\right), S\right)$.

It was pointed out in [2] that there is a natural relationship between these two definitions. Namely, a Banach frame is an atomic decomposition if and only if the unit vectors form a basis for the space $X_{d}$. Moreover, Banach frames are quite general and in fact, every Banach space has a Banach frame. Unconditional convergence plays an essential role in Banach space theory. Taking the consideration of the unconditionality, Casazza, Han and Larson in 2 gave three natural formulations of frames for a Banach space and proved that all three definitions give the same frames. The following is the one we will use in this paper.

Definition 3. A framing model is a Banach space $Z$ with a fixed unconditional basis $\left(e_{n}\right)$ for $Z$. Let $\left\{y_{n}\right\}$ be a sequence in $X^{*}$ and $S: Z \rightarrow X$ be a bounded operator. The pair $\left(\left\{y_{n}\right\}, S\right)$ is called a frame modeled on $\left(Z,\left(e_{i}\right)_{i \in \mathbb{Z}}\right)$ if the following hold:

(i) $\sum_{n}\left\langle x, y_{n}\right\rangle e_{n} \in Z, \forall x \in X$, where the convergence is unconditional.

(ii) The norms $\|x\|$ and $\left\|\sum_{n}\left\langle x, y_{n}\right\rangle e_{n}\right\|$ are equivalent.

(iii) $S\left(\sum_{n}\left\langle x, y_{n}\right\rangle e_{n}\right)=x, \forall x \in X$.

We point out that, unlike Hilbert space frames for which the reconstruction operator $S$ always exists, for Banach space frames this is not the case. That is why the existence of a reconstruction operator is required in the different definitions of Banach frames. In fact, it can be proven that the existence of such a reconstruction operator is equivalent to the property that the space $\left\{\sum_{n}\left\langle x, y_{n}\right\rangle e_{n}: x \in X\right\}$ is complemented in $Z$; see [3. The following lemma, whose proof can be found in many standard textbooks on Banach spaces, will be needed in the proof of the main theorems.

Lemma 2.1. Suppose that $\left\{x_{n}\right\}$ is an unconditional basis for a Banach space $X$. For any sequence $\left\{a_{n}\right\}$ with $\left|a_{n}\right| \leq c$, for all $n$, there exists $M>0$ such that

$$
\left\|\sum_{n} a_{n} c_{n} x_{n}\right\| \leq M\left\|\sum_{n} c_{n} x_{n}\right\|
$$

whenever $\sum_{n} c_{n} x_{n}$ exists.

\section{The SAMPLING EXPANSION}

Let $\left(\left\{y_{n}\right\}, S\right)$ be a frame modeled on $\left(Z,\left\{e_{n}\right\}\right)$ and let $P(\lambda)$ be as in (2.1). Define $L_{\lambda}$ as follows: For $\lambda \neq \lambda_{1}, \lambda_{2}, \ldots$, set

$$
L_{\lambda} x=S\left(\sum_{n} \frac{P(\lambda)}{\left(\lambda-\lambda_{n}\right)}\left\langle x, y_{n}\right\rangle e_{n}\right), \forall x \in X,
$$

while for $\lambda=\lambda_{k}, L_{\lambda_{k}} x=P^{\prime}\left(\lambda_{k}\right)\left\langle x, y_{k}\right\rangle S e_{k}, \forall x \in X$, For each $x \in X$, define $F: \mathbb{C} \rightarrow X$ by $F(\lambda)=L_{\lambda} x$. Now we state and prove the main theorem of this section.

Theorem 3.1. (1) For each fixed $\lambda \in \mathbb{C}, L_{\lambda}$ is a bounded linear operator on $X$.

(2) If $K$ is a compact subset of $\mathbb{C}$, then the set $\left\{L_{\lambda}: \lambda \in K\right\}$ is uniformly bounded. 
(3) $F(\lambda)$ is a continuous vector-valued function that is completely determined by its values $\left\{F\left(\lambda_{n}\right)\right\}$ and can be reconstructed from these values according to the formula

$$
F(\lambda)=\sum_{n} \frac{P(\lambda)}{\left(\lambda-\lambda_{n}\right) P^{\prime}\left(\lambda_{n}\right)} F\left(\lambda_{n}\right)
$$

where the convergence is unconditional.

Proof. (i) We prove (1) and (2) together, but first we show that $L_{\lambda} x$ is well-defined. Let us denote the sequence $\left\{\lambda_{n}\right\}$ by $\Lambda$. For any fixed $\lambda \neq \lambda_{1}, \lambda_{2}, \ldots$, let $d_{\lambda}=$ $\operatorname{dist}(\lambda, \Lambda)>0$. Then there exists $M_{\lambda}>0$ so that

$$
\left|\frac{P(\lambda)}{\lambda-\lambda_{n}}\right| \leq\left|\frac{P(\lambda)}{d_{\lambda}}\right|=M_{\lambda}<\infty .
$$

Since by assumption $\sum_{n}\left\langle x, y_{n}\right\rangle e_{n}$ converges unconditionally in $Z$, it follows from Lemma 2.1 that $\sum_{n} \frac{P(\lambda)}{\left(\lambda-\lambda_{n}\right)}\left\langle x, y_{n}\right\rangle e_{n} \in Z$. Thus $L_{\lambda} x$ is well-defined.

Let $K$ be a compact subset of $\mathbb{C}$ and $\Lambda_{K}=\left\{\lambda_{i_{1}}, \lambda_{i_{2}}, \cdots, \lambda_{i_{q}}\right\}$ be the set of all $\lambda_{i}$ 's that lie inside $K$. Denote the distance between $K$ and $\Lambda-\Lambda_{K}$ by $\delta$. Then for any $\lambda \in K$ and $\lambda_{k} \in \Lambda-\Lambda_{K}$, we have

$$
\sup _{\lambda \in K}\left|\frac{P(\lambda)}{\lambda-\lambda_{k}}\right| \leq \frac{1}{\delta} \sup _{\lambda \in K}|P(\lambda)|=\frac{1}{\delta}\|P\|_{K} .
$$

Set

$$
h_{i}(\lambda)=\frac{P(\lambda)}{\lambda-\lambda_{i}}, \quad i=i_{1}, i_{2}, \cdots, i_{q} .
$$

Clearly, $h_{i}$ is analytic except possibly at $\lambda=\lambda_{i}$, but since $P$ has a zero at $\lambda=$ $\lambda_{i}, h_{i}$ is actually entire. Therefore, $\max _{\lambda \in K}\left|h_{i}(\lambda)\right|=\left\|h_{i}\right\|_{K}$ is finite. Set $C=$ $\max \left\{\left\|h_{i_{1}}\right\|_{K}, \cdots,\left\|h_{i_{q}}\right\|_{K}\right\}$, and $C(K)=\max \left\{C,\|P\|_{K} / \delta\right\}$. Thus, we have shown that there exists $C(K)>0$ such that $\sup _{\lambda \in K}\left|\frac{P(\lambda)}{\lambda-\lambda_{k}}\right| \leq C(K)$ for all $k$.

Thus, by Lemma 2.1, it follows that for all $\lambda \in K$, there exists $M>0$ such that

$$
\left\|\sum_{n} \frac{P(\lambda)}{\left(\lambda-\lambda_{n}\right)}\left\langle x, y_{n}\right\rangle e_{n}\right\| \leq M\left\|\sum_{n}\left\langle x, y_{n}\right\rangle e_{n}\right\| .
$$

Also by (ii) of Definition 3 there exists a positive constant $N$ such that

$$
\left\|\sum_{n}\left\langle x, y_{n}\right\rangle e_{n}\right\| \leq N\|x\|, \forall x \in X
$$

Then from (3.2) and (3.3) we have

$$
\begin{aligned}
\left\|L_{\lambda} x\right\| & =\left\|S\left(\sum_{n} \frac{P(\lambda)}{\left(\lambda-\lambda_{n}\right)}\left\langle x, y_{n}\right\rangle e_{n}\right)\right\| \\
& \leq\|S\|\left\|\sum_{n} \frac{P(\lambda)}{\left(\lambda-\lambda_{n}\right)}\left\langle x, y_{n}\right\rangle e_{n}\right\| \\
& \leq M\|S\|\left\|\sum_{n}\left\langle x, y_{n}\right\rangle e_{n}\right\| \leq M N\|S\|\|x\| .
\end{aligned}
$$

So $\left\|L_{\lambda}\right\| \leq\|S\| M N$ for any $\lambda \in K$, i.e., $\left\|L_{\lambda}\right\|$ is uniformly bounded on $K$.

(ii) To prove that $F(\lambda)$ is continuous, we show that $G(\lambda)=\frac{1}{P(\lambda)} F(\lambda)$ is continuous and since $P(\lambda)$ is entire, it will follow that $F$ is continuous. Let $\lambda \notin \Lambda$, and 
$d_{\lambda}$ be the distance from $\lambda$ to $\{\Lambda\}$. Then, for any $\mu \in \mathbb{C}$ such that $|\lambda-\mu|<d_{\lambda} / 2$, we have

$$
\frac{1}{\left|\lambda-\lambda_{n}\right|\left|\mu-\lambda_{n}\right|} \leq 2 d_{\lambda}^{-2}
$$

Hence, by Lemma 2.1 again, there is a constant $M>0$ so that

$$
\left\|\sum_{n} \frac{1}{\left(\lambda-\lambda_{n}\right)\left(\mu-\lambda_{n}\right)}\left\langle x, y_{n}\right\rangle e_{n}\right\| \leq M\left\|\sum_{n}\left\langle x, y_{n}\right\rangle e_{n}\right\|
$$

for all $\nu$ such that $|\mu-\lambda|<d_{\lambda} / 2$. Therefore, once more from (3.2) and (3.3) we have

$$
\begin{aligned}
\|G(\lambda)-G(\mu)\| & =|\lambda-\mu|\left\|S\left(\sum_{n} \frac{1}{\left(\lambda-\lambda_{n}\right)\left(\mu-\lambda_{n}\right)}\left\langle x, y_{n}\right\rangle e_{n}\right)\right\| \\
& \leq M|\lambda-\mu|\|S \mid\|\left\|\sum_{n}\left\langle x, y_{n}\right\rangle e_{n}\right\| \\
& \leq M N|\lambda-\mu|\|S\|\|x\|,
\end{aligned}
$$

which implies that $\lim _{\mu \rightarrow \lambda} G(\mu)=G(\lambda)$. Moreover, for $\lambda=\lambda_{k}$, we clearly have $\lim _{\mu \rightarrow \lambda_{k}} F(\mu)=P^{\prime}\left(\lambda_{k}\right)\left\langle x, y_{k}\right\rangle S e_{k}=F\left(\lambda_{k}\right)$. From this and the continuity of $F(\lambda)$, Equation (3.1) follows.

We close this section by two examples demonstrating the main result.

Example 3.1. Let $X=L^{p}[-\pi, \pi]$ for $1<p \leq 2$. Then $X^{*}=L^{q}[-\pi, \pi]$ where $1 / p+1 / q=1$. It is known 12 that if $\left\{\lambda_{n}\right\}$ is a sequence of real numbers so that $\left|\lambda_{n}-n\right| \leq(p-1) / 2 p$ for all $n$, then $\left\{e^{i \lambda_{n} t}: n \in \mathbb{Z}\right\}$ is an unconditional basis for $L^{p}[-\pi, \pi]$. Thus, there is a sequence $\left\{h_{n}: n \in \mathbb{Z}\right\} \subset L^{q}[-\pi, \pi]$ such that

$$
f=\sum_{n \in \mathbb{Z}}\left\langle f, h_{n}\right\rangle e^{i \lambda_{n} t},
$$

for any $f \in L^{p}[-\pi, \pi]$, where the convergence is unconditional. Now let

$$
Z=\left\{\left\{c_{n}\right\}: \sum_{n} c_{n} e^{i \lambda_{n} t} \text { converges unconditionally in } L^{p}[-\pi, \pi]\right\},
$$

equipped with the norm $\left\|\left\{c_{n}\right\}\right\|=\left\|\sum_{n} c_{n} e^{i \lambda_{n} t}\right\|$. Then $\left\{e_{n}\right\}$ will be an unconditional Riesz basis for $Z$, where $e_{n}(m)=\delta_{n, m}$. Thus $S: Z \rightarrow L^{p}[-\pi, \pi]$ defined by $S\left(\left\{c_{n}\right\}\right)=\sum_{n} c_{n} e^{i \lambda_{n} t}$ is a bounded operator such that $S\left(\sum_{n}\left\langle f, h_{n}\right\rangle e_{n}\right)=$ $\sum_{n}\left\langle f, h_{n}\right\rangle e^{i \lambda_{n} t}=f$ for all $f \in L^{p}[-\pi, \pi]$. Therefore Theorem 2.1 applies and we have: if

$$
F(\lambda)=S\left(\sum_{n} \frac{P(\lambda)\left\langle f, h_{n}\right\rangle}{\left(\lambda-\lambda_{n}\right)} e_{n}\right)=\left(\sum_{n} \frac{P(\lambda)\left\langle f, h_{n}\right\rangle}{\left(\lambda-\lambda_{n}\right)} e^{i \lambda_{n} t}\right)
$$

then

$$
F(\lambda)=\sum_{n} \frac{P(\lambda)}{\left(\lambda-\lambda_{n}\right) P^{\prime}\left(\lambda_{n}\right)} F\left(\lambda_{n}\right),
$$

where the series converges in $L^{p}[-\pi, \pi]$.

Example 3.2. Let $W:[0, \infty) \rightarrow \mathbb{R}^{+}$be a radially decreasing function, i.e., $W$ is decreasing, with $W(0)<\infty$, and belongs to $L^{1}\left(\mathbb{R}^{+}\right)$. We say that $W$ is an $L^{1}$-majorant of a function $g$ defined on $\mathbb{R}$ if $|g(x)| \leq W(|x|)$. Let $\psi$ be an 
orthonormal wavelet such that $\psi$ and $\psi^{\prime}$ have a common radial decreasing $L^{1}$ majorant $W$ satisfying $\int_{0}^{\infty} s W(s) d s<\infty$. Then it is known [14 that the system of wavelets $\left\{\psi_{j, k}(x)=2^{j / 2} \psi\left(2^{j} x-k\right), j, k \in \mathbb{Z}\right\}$ is an unconditional basis for $L^{p}(\mathbb{R}), 1<p<\infty$.

Let $X=L^{p}(\mathbb{R})$ for $1<p<\infty$. Then $X^{*}=L^{q}(\mathbb{R})$, where $1 / p+1 / q=1$. The set $\left\{\psi_{j, k}(x)\right\}$ is also an unconditional basis for $L^{q}(\mathbb{R})$. Therefore, we have

$$
f(x)=\sum_{j, k \in \mathbb{Z}}\left\langle f, \psi_{j, k}\right\rangle \psi_{j, k}(x)
$$

for any $f \in L^{p}(\mathbb{R})$, where the convergence is unconditional.

Now let us map $\mathbb{Z} \times \mathbb{Z}$ one-to-one into $\mathbb{Z}$ and set

$$
Z=\left\{\left\{c_{j, k}\right\}: \sum_{j, k} c_{j, k} \psi_{j, k}(x) \text { converges unconditionally in } L^{p}(\mathbb{R})\right\}
$$

and equip it with the norm $\left\|\left\{c_{j, k}\right\}\right\|=\left\|\sum_{j, k} c_{j, k} \psi_{j, k}\right\|$. It follows that $\left\{e_{n}\right\}$ is an unconditional Riesz basis for $Z$. Thus $S: Z \rightarrow L^{p}(\mathbb{R})$ defined by $S\left\{c_{j, k}\right\}=$ $\sum_{j, k} c_{j, k} \psi_{j, k}$ is a bounded operator such that

$$
S\left(\sum_{j, k}\left\langle f, \psi_{j, k}\right\rangle e_{n}\right)=\sum_{j, k}\left\langle f, \psi_{j, k}\right\rangle \psi_{j, k}=f
$$

for all $f \in L^{p}(\mathbb{R})$. Therefore, by Theorem 3.1 it follows that if

$$
F(\lambda)=S\left(\sum_{j, k} \frac{P(\lambda)\left\langle f, \psi_{j, k}\right\rangle}{\left(\lambda-\lambda_{n}\right)} e_{n}\right),
$$

where $n$ is that unique integer that corresponds to the pair $(j, k)$, then

$$
F(\lambda)=\sum_{n} \frac{P(\lambda)}{\left(\lambda-\lambda_{n}\right) P^{\prime}\left(\lambda_{n}\right)} F\left(\lambda_{n}\right),
$$

where the series converges in $L^{p}(\mathbb{R})$.

\section{INVERSION FORMULA}

Now we derive an inversion formula for the vector-valued function $F(\lambda)$ using Bochner integrals.

Theorem 4.1. Let $Z=\ell^{p}, 1<p \leq 2$, and let $\left\{\lambda_{n}\right\}_{n \in \mathbb{Z}}$ be a sequence of real numbers having $\pm \infty$ as its only limit points and no finite limit point such that

$$
\sup _{n \in \mathbb{Z}}\left|\lambda_{n}-n\right| \leq C<\frac{(p-1)}{2 p}, \quad-\infty<n<\infty,
$$

for some $p, 1<p \leq 2$. Let

$$
P(\lambda)=\left(\lambda-\lambda_{0}\right) \prod_{n=1}^{\infty}\left(1-\frac{\lambda}{\lambda_{n}}\right)\left(1-\frac{\lambda}{\lambda_{-n}}\right),
$$

with

$$
\int_{-\infty}^{\infty}\left|\frac{P(\lambda)}{\lambda-\lambda_{n}}\right|^{p} \leq D<\infty, \quad \forall n
$$


Then if

$$
F(\lambda)=S\left(\sum_{n} \frac{P(\lambda)}{\left(\lambda-\lambda_{n}\right)}\left\langle x, y_{n}\right\rangle e_{n}\right), \quad x \in X
$$

we have

$$
x=\lim _{N \rightarrow \infty} \int_{-\infty}^{\infty} F(\lambda) K_{N}(\lambda) d \lambda
$$

where

$$
K_{N}(\lambda)=\sum_{k=-N}^{N} \frac{B_{k}(\lambda)}{P^{\prime}\left(\lambda_{k}\right)} \text { and } \quad B_{k}(\lambda)=\frac{1}{2 \pi} \int_{-\sigma}^{\sigma} e^{i \lambda_{k} t} e^{-i \lambda t} d t .
$$

Proof. From Theorem 18, p. 48 and Lemma 16.2, p. 57 in 12, we infer that there exists a sequence of functions $\left\{h_{n}(x)\right\} \subset L^{q}(-\pi, \pi)$, where $1 / p+1 / q=1$ such that

$$
\frac{P(\lambda)}{\left(\lambda-\lambda_{n}\right) P^{\prime}\left(\lambda_{n}\right)}=\int_{-\sigma}^{\sigma} h_{n}(t) e^{i \lambda t} d t
$$

It is easy to see that $B_{k}(\lambda)$ is in $L^{p}(\mathbb{R})$ for all $1<p \leq \infty$, from which it easily follows that

$$
\int_{-\infty}^{\infty} \frac{P(\lambda) B_{k}(\lambda)}{\left(\lambda-\lambda_{n}\right) P^{\prime}\left(\lambda_{n}\right)} d \lambda=\int_{-\sigma}^{\sigma} h_{n}(t) e^{i \lambda_{k} t} d t=\delta_{n, k}
$$

The integral on the left-hand side converges absolutely, in view of Hölder's inequality, because $P(\lambda) /\left(\lambda-\lambda_{k}\right) \in L^{p}(\mathbb{R})$ for $1<p \leq 2$ and $B_{k}(\lambda) \in L^{q}(\mathbb{R})$ for $1<q \leq \infty$, in particular for $2 \leq q \leq \infty$. Thus,

$$
\begin{aligned}
\int_{-\infty}^{\infty} F(\lambda) K_{N}(\lambda) d \lambda & =\int_{-\infty}^{\infty} \sum_{n=1}^{\infty} \frac{P(\lambda) F\left(\lambda_{n}\right)}{\left(\lambda-\lambda_{n}\right) P^{\prime}\left(\lambda_{n}\right)} \cdot \sum_{k=-N}^{N} \frac{B_{k}(\lambda)}{P^{\prime}\left(\lambda_{k}\right)} d \lambda \\
& =\sum_{n=1}^{\infty} \sum_{k=-N}^{N} \frac{F\left(\lambda_{n}\right)}{P^{\prime}\left(\lambda_{k}\right)} \cdot \int_{-\infty}^{\infty} \frac{P(\lambda) B_{k}(\lambda)}{\left(\lambda-\lambda_{n}\right) P^{\prime}\left(\lambda_{n}\right)} d \lambda \\
& =\sum_{n=1}^{\infty} \sum_{k=-N}^{N} \frac{F\left(\lambda_{n}\right)}{P^{\prime}\left(\lambda_{k}\right)} \delta_{n, k}=\sum_{n=-N}^{N} \frac{F\left(\lambda_{n}\right)}{P^{\prime}\left(\lambda_{n}\right)} \\
& =\sum_{n=-N}^{N} \frac{P^{\prime}\left(\lambda_{n}\right)\left\langle x, y_{n}\right\rangle}{P^{\prime}\left(\lambda_{n}\right)} S e_{n}=\sum_{n=-N}^{N}\left\langle x, y_{n}\right\rangle S e_{n} .
\end{aligned}
$$

Hence, by the continuity of $S$ it follows that

$$
\lim _{N \rightarrow \infty} \int_{-\infty}^{\infty} F(\lambda) K_{N}(\lambda) d \lambda=\lim _{N \rightarrow \infty} S\left(\sum_{n=-N}^{N}\left\langle x, y_{n}\right\rangle e_{n}\right)=x .
$$

The proof will be complete if we can justify interchanging the integration and the infinite summation above.

Because $B_{k} \in L^{q}(\mathbb{R})$, so is $K_{N}$; therefore, by the Lebesgue dominated convergence theorem, it suffices to show that $\int_{-\infty}^{\infty}\|F(\lambda)\|^{p} d \lambda<\infty$. But first, we need to show that for all $y \in X^{*}$ with $\|y\|=1$,

$$
\sup _{\|y\|=1}\left(\sum_{n}\left|\left\langle S e_{n}, y\right\rangle\right|^{q}\right)^{1 / q}<\infty .
$$


Let $e_{n}$ be the standard basis for $l^{p}$. Then for any $\eta=\left(\eta_{n}\right) \in l^{p}$, we have $\eta=$ $\sum_{n} \eta_{n} e_{n}$. Now for any $y \in X^{*}$, we have $S^{*} y \in Z^{*}=l^{q}$. However, $\left\langle\eta, S^{*} y\right\rangle=$ $\langle S \eta, y\rangle=\left\langle\sum_{n} \eta_{n} S e_{n}, y\right\rangle=\sum_{n} \eta_{n}\left\langle S e_{n}, y\right\rangle$ holds for all $l^{p}$ sequence $\left(\eta_{n}\right)$. Therefore $\left(\left\langle S e_{n}, y\right\rangle\right)$ must be an $l^{q}$-sequence which determines the bounded linear functional $S^{*} y$.

But since $\left\|S^{*} y\right\|=\left(\sum_{n}\left|\left\langle S e_{n}, y\right\rangle\right|^{q}\right)^{1 / q}$ and $S^{*}$ is a bounded linear operator, we have

$$
\sup _{\|y\|=1}\left\|S^{*} y\right\|=\sup _{\|y\|=1}\left(\sum_{n}\left|\left\langle S e_{n}, y\right\rangle\right|^{q}\right)^{1 / q} \leq\left\|S^{*}\right\|<\infty .
$$

Now since $\|F(\lambda)\|=\sup _{\|y\|=1}|\langle F(\lambda), y\rangle|, \quad$ for all $y \in X^{*}$ with $\|y\|=1$, it follows that

$$
\|F(\lambda)\|=\sup _{\|y\|=1}\left|\sum_{n} \frac{P(\lambda)}{\left(\lambda-\lambda_{n}\right)}\left\langle x, y_{n}\right\rangle\left\langle S e_{n}, y\right\rangle\right| .
$$

By using Hölder's inequality and (4.2), we obtain

$$
\begin{aligned}
\|F(\lambda)\| & \leq\left(\sum_{n}\left|\frac{P(\lambda)}{\lambda-\lambda_{n}}\right|^{p}\left|\left\langle x, y_{n}\right\rangle\right|^{p}\right)^{1 / p} \sup _{\|y\|=1}\left(\sum_{n}\left|\left\langle S e_{n}, y\right\rangle\right|^{q}\right)^{1 / q} \\
& \leq\left(\sum_{n}\left|\frac{P(\lambda)}{\lambda-\lambda_{n}}\right|^{p}\left|\left\langle x, y_{n}\right\rangle\right|^{p}\right)^{1 / p}\left\|S^{*}\right\|,
\end{aligned}
$$

which, in view of (4.1), yields

$$
\int_{-\infty}^{\infty}\|F(\lambda)\|^{p} d \lambda \leq D\left(\sum_{n}\left|\left\langle x, y_{n}\right\rangle\right|^{p}\right)\left\|S^{*}\right\|^{p}<\infty .
$$

This completes the proof.

\section{REFERENCES}

[1] M.H. Annaby and A.I. Zayed, On the use of Green's function in sampling theory, J. Integral Equations and Applications, 10 (1998), 117-139. MR1646834 (99g:34061)

[2] P. Casazza, D. Han and D. Larson, Frames for Banach spaces, Contemp. Math., 247, Amer. Math. Soc., Providence, RI, 1999, 149-182. MR.1738089 (2000m:46015)

[3] O. Christensen and D. Stoeva, $p$-frames in separable Banach spaces, Adv. Comput. Math., 18 (2003), 117-126. MR.1968115 (2004b:42060)

[4] O. Christensen, Atomic decomposition via projective group representations, Rocky Mountain J. Math., 26 (1996), 1289-1312. MR1447588 (98h:43004)

[5] O. Christensen and C. Heil, Perturbations of Banach frames and atomic decompositions, Math. Nachr 185 (1997), 33-47. MR1452474 (98m:42061)

[6] H. Feichtinger, Atomic characterizations of modulation spaces through Gabor-type representations, Rocky Mountain J. Math., 19 (1989), 113-125. MR1016165 (90h:46051)

[7] H. Feichtinger and K. Gröchenig, Banach spaces related to integrable group representations and their atomic decompositions I, J. Funct. Anal., 86 (1989), 307-340. MR1021139 (91g:43011)

[8] H. Feichtinger and K. Gröchenig, Banach spaces related to integrable group representations and their atomic decompositions II, Monatsh. Math., 108 (1989), 129-148. MR.1026614 (91g:43012)

[9] K. Gröchenig, Describing functions: Atomic decompositions versus frames, Monatshefte fur Math., 112(1991), 1-42. MR1122103 (92m:42035)

[10] M. Frazier and B. Jawerth, Decomposition of Besov Spaces, Indiana Univ. Math. J., 34 (1985), 777-799. MR0808825 (87h:46083)

[11] H.P. Kramer, A generalized sampling theorem, J. Math. Phys. 38 (1959), 68-72. MR0103786 $(21: 2550)$ 
[12] N. Levinson, Gap and Density Theorems, Amer. Math. Soc. Colloq. Publs. Ser., Vol. 26, 1940. MR0003208(2:180d)

[13] M.A. Naimark, Linear Differential Operators I, George Harrap, London, 1967.

[14] G. Weiss and E. Hernandez, A First Course On Wavelets, CRC Press, Boca Raton, FL 1996. MR.1408902 (97i:42015)

[15] A. Zayed, Sampling in a Hilbert space, Proc. Amer. Math. Soc., 124 (1996), 3767-3776. MR.1343731 (97b:41007)

[16] A.I. Zayed, A new role of Green's function in interpolation and sampling theory, J. Math. Anal. Appl. 175 (1993), 222-238. MR1216757 (94d:41011)

[17] A.I. Zayed, Advances in Shannon's Sampling Theory, CRC Press, Boca Raton, 1993. MR:1270907 (95f:94008)

Department of Mathematics, University of Central Florida, Orlando, Florida 32816

E-mail address: dhan@pegasus.cc.ucf.edu

Department of Mathematical Sciences, DePaul University, Chicago, Illinois 60614

E-mail address: azayed@math.depaul.edu 